\title{
Article
}

\section{Optimal Load and Energy Management of Aircraft Microgrids Using Multi-Objective Model Predictive Control}

\author{
Xin Wang ${ }^{1}$, Jason Atkin ${ }^{2}$, Najmeh Bazmohammadi ${ }^{3}$, Serhiy Bozhko ${ }^{1}$, and Josep M. Guerrero ${ }^{3, *}$ \\ 1 Department of Electrical and Electronic Engineering, Faculty of Engineering, University of Nottingham; \\ \{xin.wang2, serhiy.bozhko\}@nottingham.ac.uk \\ 2 Computational Optimisation and Learning Lab, School of Computer Science, University of Nottingham; jason.at- \\ kin@nottingham.ac.uk \\ 3 Centre for Research on Microgrids (CROM), AAU Energy, Aalborg University; \{naj, joz\}@energy.aau.dk \\ * Correspondence: joz@energy.aau.dk
}

\begin{abstract}
Safety issues related to the electrification of more electric aircraft (MEA) need to be addressed because of the increasing complexity of aircraft electrical power systems and the growing number of safety-critical sub-systems that need to be powered. Managing the energy storage systems and the flexibility in the load-side plays an important role in preserving the system's safety when facing an energy shortage. This paper presents a system-level centralized operation management strategy based on model predictive control (MPC) for MEA to schedule battery systems and exploit flexibility in the demand-side while satisfying time-varying operational requirements. The proposed online control strategy aims to maintain energy storage (ES) and prolong the battery life cycle, while minimizing load shedding, with fewer switching activities to improve devices lifetime and to avoid unnecessary transients. Using a mixed-integer linear programming (MILP) formulation, different objective functions are proposed to realize the control targets, with soft constraints improving the robustness of the model. Besides, an evaluation framework is proposed to analyze the effects of various objective functions and the prediction horizon on system performance, which provides the designers and users of MEA and other complex systems with new insights into operation management problem formulation.
\end{abstract}

Keywords: Model predictive control; Mixed-integer linear programming; Multi-objective optimization; Energy storage management; Load management; More electric aircraft; Demand-side flexibility

\section{Introduction}

Onboard power demands increase dramatically with the development of more electric aircraft (MEA) and the replacement of traditional hydraulics and pneumatics by electrical systems to achieve higher efficiency and less fuel burn [1]. Critical loads, which are responsible for flight safety, such as flight surface actuators and environmental control systems, must be powered in all flight scenarios regardless of high power peaks or faultcausing emergencies. The energy storage system (ESS) has an important role in MEA, not only for supporting bus voltages, but also as a backup to deal with power shortages during faults occurring in generation or transmission systems, or high power peaks. In addition, shedding of non-critical loads (e.g. passenger cabin lighting) is also used to reduce peak power usage, helping to maintain power for safety-critical loads [2]. Intelligently combining the load shedding with the ESS capabilities is essential for the safe onboard electrical power system (EPS) operation.

Due to the complexity of MEA optimal operation management and the importance of their safety and reliability resulting from an advanced operating strategy, considerable 
attention is being devoted to developing suitable models and algorithms. Finite state machines are adopted in [3], [4], and [5] to model the onboard DC-based EPS of MEA as a set of states and transitions. In these studies, several sets of safety rules are defined to cope with different failure cases to ensure uninterrupted power supply to the high priority loads. However, this method relies on accurate measurements to change the operation state and it is hard to integrate multiple operating goals. In addition, setting optimal rules can be difficult for complex systems because of the large number of system states.

Heuristic and metaheuristic algorithms are also widely used to solve energy and load management problems for microgrids (MGs), such as fuzzy logic algorithm [6], [7], [8], [9], genetic algorithms [10], [11], [12], particle swarm optimization [13], [14], [15], and evolutionary algorithms [16], [17]. However, these approaches are not suitable for real-time application, since they might fall into the trap of suboptimal solutions and can still be computationally intensive. Neural networks, studied in [18], [19], are fast but need training, hence are less capable of coping with unexpected situations, and it can be difficult to verify their reliability for aircraft usage.

Recently, Model Predictive Control (MPC) has drawn the attention of the power system community for the control and operation management of MGs. MPC provides an optimal operating strategy based on predicted future system trajectories, thereby avoiding operating based on the worst-case scenarios with high costs. The inherent feedback mechanism of MPC stemming from its receding horizon mechanism makes the system more robust against uncertainty [20]. In [21], [22], an MPC-based hierarchical control structure is applied for achieving economic efficiency in residential MGs. In [23], [24], MPC supervisory control is used to compensate for the mismatch between the offline scheduling process and the real-time operation of a hybrid PV-wind-battery MG. In this research, the system models are formulated as Mixed-Integer Linear Programming (MILP) problems. The MPC method has also been adopted for battery management in Hybrid Electric Vehicles (HEV) in [25], [26], in which the MPC controller attempts to minimize the vehicle equivalent fuel consumption and the battery capacity fade at the same time. To enhance the battery lifespan and power performance, [27] and [28] propose an MPC approach to reduce the battery power variation and maintain supercapacitors' SOC at their desired values. In [29], the battery capacity fading is described by an online leastsquares identification method, and the degradation cost function is used in the MPC scheme to increase the battery lifetime. Since MPC has shown good performance for residential MGs and HEVs energy management, researchers have been explored the possibility of its application in MEA EPS. In the application for MEA, on the one hand, the robustness of controller design can benefit from the intrinsic robust characteristics of MPC due to the rolling horizon strategy. On the other hand, the system operational and time-coupling constraints can be integrated into the MPC model. In [30], a hierarchical MPC framework for a hybrid propulsion system is presented to minimize power generation costs and delivery losses. However, the possibility of load shedding and system failure is not considered. In [31], a rate-based MPC method is adopted for power sharing between two generators that are driven by the same engine. The optimal energy management based on MPC by sharing power among different power sources for hybrid propulsion system onboard is studied in [32]. In [33] and [34], MPC is used for both power scheduling and load shedding optimization to cope with scenarios with one generator and one transmission cable failure. However, in these papers, the MPC for battery management is not the focus and the control problem is formulated without much detail about, for example, how to cope with different control targets and increase algorithm robustness, which has left considerable scope for further investigation and analysis here. Hence, this paper aims to address these issues in the proposed MPC strategy for MEA EPS.

In the aforementioned works of the MPC applications, the flexibility of the MPC design including the objective function formulation, prediction horizon length, and the weighting factors to prioritize different operating criteria and the related impacts to the 
system performance has been neglected by adopting empirical settings. In fact, investigating how these degrees of freedom can affect the control system performance will help to better decide the settings of MPC to meet system requirements. This paper will contribute to this investigation by designing a system-level MPC-based operation management strategy for a MEA. The analysis in this study can be extended to other complex systems including storage systems and load management among their targets.

In the studied system, the MPC controller aims to keep the battery energy storage highly charged, with fewer power variations to prolong the battery life cycle, and to minimize load shedding, with less contactor switching. Organizing these operating criteria in the form of an appropriate objective function is essential for MPC optimization models. However, the flexibility in the formulation of the objective functions adds to the complexity of the design.

In this paper, to solve the multi-objective optimal operation management problem of the MEA EPS, a MPC-based operation management strategy is introduced targeting on intelligently managing the energy storage systems onboard and exploiting flexibility in the demand-side while satisfying time-varying operational requirements. Different objective functions are proposed with various cost function terms to reach the MEA operating goals, where each objective function can perform differently for each control target. To evaluate the designed control system performance, an evaluation index for each target is proposed to quantify system performance for overall flight stages. All objective functions are evaluated by the proposed indices with the prediction horizon changing from short to long; hence, the impact of various prediction horizons for each objective function can be compared. This evaluation procedure is conducted with the load profiles having different changing frequencies and variations, so that the analysis can consider different load cases. Based on the proposed index for each target, a multi-objective evaluation index is also proposed as an overall performance criterion, by which the final selection of the objective function and the length of the prediction horizon is performed.

In addition to analysis of the degrees of freedom in an MPC setting, this work also improves the robustness of the MPC-based control strategy to adapt the controller to realtime operating conditions. On the one hand, soft constraints are introduced to cope with the non-accurate estimation of the battery SOC. The optimization problem to be solved by the control system is formulated as a MILP problem, which includes both objective functions and system constraints related to the system power flow, energy conversion, and connection constraints, benefitting from certainty for global optimal solutions [35]. As the system only operates normally when the MILP-MPC algorithm provides a feasible solution, the algorithm should be robust enough to tolerate bounds exceeding situations (i.e. SOC exceeds the target range). On the other hand, the real-time application requires that the sample time of the MPC can meet the possible computation time variations when dealing with different operating conditions. For each objective function, the worst-case values of the maximum computation time are recorded for various prediction horizon lengths. The sample time can be selected appropriately by considering the maximum computation time at the design stage, which guarantees that the MPC can be adopted for online optimization in real-time. Finally, a hardware-in-the-loop (HIL) experiment is conducted for the studied system, to verify the real-time application of the proposed MPC-based operation management strategy.

The rest of this paper is organized as follows: Section 2 introduces the MEA system under consideration while the proposed MILP-MPC controller framework is introduced in Section 3. The system model with different objective functions is proposed in Section 4. In Section 5, the evaluation framework is set up, based on which, the performance for each objective function is evaluated with the changing prediction horizon for two different load profiles. The real-time analysis regarding the sample time selection is also presented in this section. The experiment setup and the results are presented in Section 6. Finally, conclusions are drawn in Section 7. 


\section{System Description}

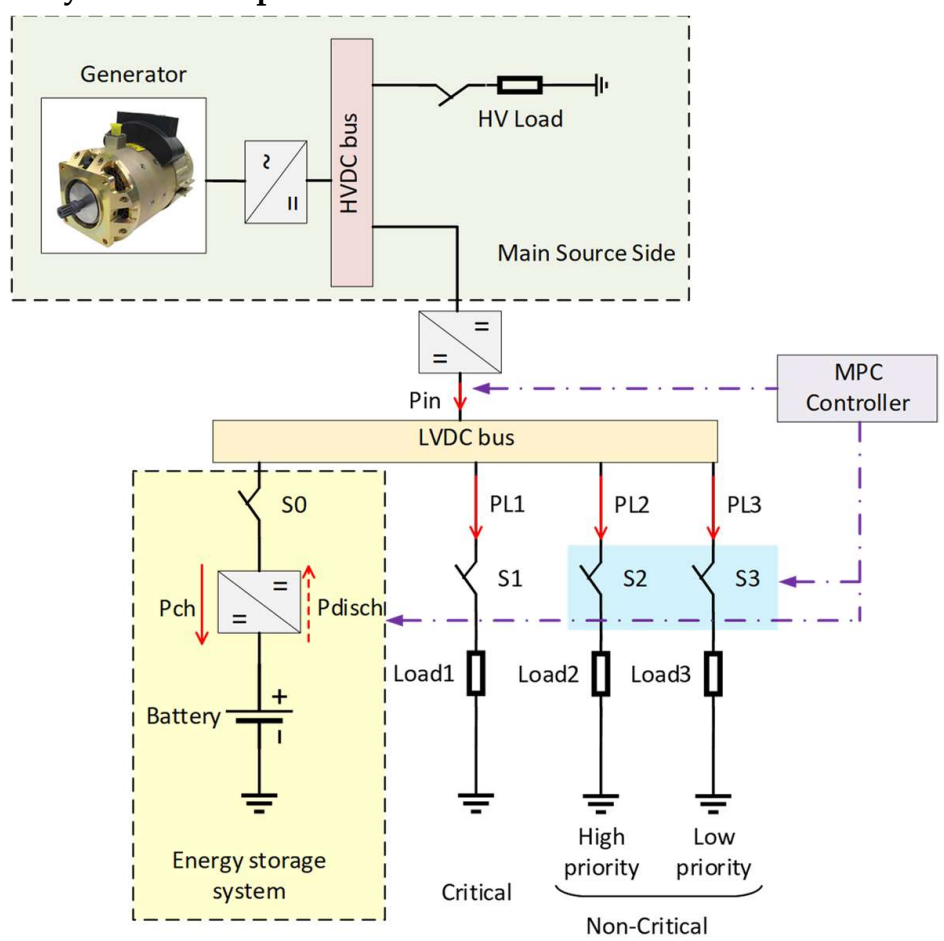

Figure 1. The architecture of EPS under study

The EPS on the aircraft are normally symmetrically allocated for the left and right sides [36]. Figure 1 presents the architecture for one side of EPS studied in this work, and Table 1 listed all the main symbols used in this paper. As can be seen in this figure, the EPS includes a main source (MS) consisting of one generator connected to an HV bus, an ESS, DC/DC power converters, and several loads. The MS powers the loads connected to a low voltage (LV) bus. In this system, the ES, which consists of one battery, one bidirectional DC/DC converter, and one contactor, is connected to the same LV bus, to both maintain the bus voltage and supply the loads when faults result in insufficient power available from the MS.

Table 1 Nomenclature

\begin{tabular}{llll}
\hline \hline Parameters & & \multicolumn{2}{l}{ Cost function terms } \\
\hline$k$ & Time intervals, $\mathrm{k} \in \mathbb{Z} \geq 0$ & $J_{S L i}$ & $\begin{array}{l}\text { Cost function for minimizing total load } \\
\text { shedding }\end{array}$ \\
\hline$H$ & Prediction horizon [s] & $J_{s L i}$ & $\begin{array}{l}\text { Cost function for minimizing switching } \\
\text { activities }\end{array}$ \\
\hline$T_{s}$ & Sampling time [s] & $J_{P c h}$ & $\begin{array}{l}\text { Cost function for maximining charging } \\
\text { power }\end{array}$ \\
\hline$T$ & Total simulation time [s] & $J_{P d i s c h}$ & $\begin{array}{l}\text { Cost function for minimizing discharging } \\
\text { power }\end{array}$ \\
\hline$\eta_{c h /} / \eta_{\text {disch }}$ & $\begin{array}{l}\text { Battery charging/discharging } \\
\text { efficiency }\end{array}$ & $J_{\Delta}$ & $\begin{array}{l}\text { Cost function for keeping SOC within the } \\
\text { target range }\end{array}$ \\
\hline$B_{c a p}$ & Battery capacity [kWh] & $J_{S O C}$ & $\begin{array}{l}\text { Cost function for maximizing SOC to the } \\
\text { upper bound }\end{array}$ \\
\hline$P_{c h}^{\max }$, & $\begin{array}{l}\text { Maximum } \\
\text { charging/discharging power }\end{array}$ & $J_{\text {Pbatt }}$ & $\begin{array}{l}\text { Cost function for minimizing battery power } \\
\text { variations }\end{array}$ \\
\hline \hline
\end{tabular}




\begin{tabular}{|c|c|c|c|}
\hline $\mathrm{LO} / \mathrm{HI}$ & Lower/upper bounds of the & \multicolumn{2}{|c|}{ Weighting factors } \\
\hline$P_{i n}^{\max }$ & $\begin{array}{l}\text { Maximum input power from } \\
\text { the MS-side [kW] }\end{array}$ & ws & Weighting factor for $J_{S L i}$ \\
\hline$P_{L i}^{\text {shed }}(k)$ & $\begin{array}{l}\text { The ith non-critical load } \\
\text { power }[\mathrm{kW}]\end{array}$ & wo & Weighting factor for $J \delta L i$ \\
\hline$\gamma_{L i}$ & $\begin{array}{l}\text { The priority of the ith non- } \\
\text { critical load }\end{array}$ & wPch & Weighting factor for $J P c h$ \\
\hline$N_{L i}$ & $\begin{array}{l}\text { Total number of non-critical } \\
\text { loads }\end{array}$ & WPdisch & Weighting factor for JPdisch \\
\hline$P_{L i}^{\text {nonshed }}(k)$ & $\begin{array}{l}\text { The ith critical load power } \\
{[\mathrm{kW}]}\end{array}$ & $w_{\Delta}$ & Weighting factor for $J_{\Delta}$ \\
\hline \multicolumn{2}{|c|}{ Continues Variables } & wSOC & Weighting factor for $J S O C$ \\
\hline$P_{\text {in }}(k)$ & $\begin{array}{l}\text { Input power from the MS- } \\
\text { side }[\mathrm{kW}]\end{array}$ & wPbatt & Weighting factor for JPbatt \\
\hline$P_{c h}(k)$ & Battery charging power [kW] & \multicolumn{2}{|c|}{ Evaluation Indices } \\
\hline$P_{\text {disch }}(k)$ & $\begin{array}{l}\text { Battery discharging power } \\
{[\mathrm{kW}]}\end{array}$ & GsLi & Evaluation index for total load shedding \\
\hline$P_{\text {batt }}(k)$ & Battery overall power $[\mathrm{kW}]$ & GsLi & $\begin{array}{l}\text { Evaluation index for contactor status } \\
\text { change }\end{array}$ \\
\hline$S O C(k)$ & Battery state of charge & Gsoc & $\begin{array}{l}\text { Evaluation index for battery energy storage } \\
\text { level }\end{array}$ \\
\hline$\varepsilon(k)$ & $\begin{array}{l}\text { Tolerance for lower bound of } \\
\text { battery SOC }\end{array}$ & Gpbatt & $\begin{array}{l}\text { Evaluation index for battery power } \\
\text { variations }\end{array}$ \\
\hline$\vartheta(k)$ & $\begin{array}{l}\text { Tolerance for upper bound of } \\
\text { battery SOC }\end{array}$ & $\begin{array}{l}G_{\overline{S O C R}} \\
G_{\underline{S O C R}}\end{array}$ & $\begin{array}{l}\text { Evaluation index for SOC target range: } \\
\text { upper and lower range bound } \\
\text { correspondingly }\end{array}$ \\
\hline \multicolumn{2}{|c|}{ Binary Variables } & \multicolumn{2}{|c|}{ Weighting factors for multi-objective evaluation index } \\
\hline$S_{L i}(k)$ & $\begin{array}{l}\text { Contactor connection status } \\
\text { of the } i \text { th non-critical load }\end{array}$ & $v s$ & Weight for GsLi \\
\hline$\zeta_{c h}(k)$ & $\begin{array}{l}\text { Indicator for charging the } \\
\text { battery }\end{array}$ & $v_{\delta}$ & Weight for G $\delta L i$ \\
\hline \multirow[t]{3}{*}{$\zeta_{\text {disch }}(k)$} & $\begin{array}{l}\text { Indicator for discharging the } \\
\text { battery }\end{array}$ & vSOC & Weight for Gsoc \\
\hline & & VPbatt & Weight for GPbatt \\
\hline & & $\begin{array}{l}v_{\overline{S O C R}} \\
v_{\underline{S O C R}}\end{array}$ & Weight for $G_{\overline{S O C R}}, G_{\underline{S O C R}}$ correspondingly \\
\hline
\end{tabular}

The MEA loads can be classified into critical and non-critical loads. Critical loads link with flight safety status and avionics systems, such as environment control system and flight control system, etc. The noncritical loads are the other classes of loads that provide a comfortable environment for passengers and some of them are more essential which are evaluated with a higher priority. For example, the in-flight entertainment and galley services are considered as a lower priority load than other non-critical loads such as water and waste [37], [38]. Therefore, in this study, three types of load are considered, namely critical loads (Load1) which must be supplied under all circumstances, high priority loads (Load2), which can be shed but should not where possible, and low priority loads (Load3), which should be shed in preference to high priority loads when load shedding is needed.

The control system is responsible for optimizing the control commands related to the MS $\left(P_{i n}\right)$ and ES $\left(P_{b a t t}\right)$ as well as determining the connection/disconnection status of noncritical loads $\left(S_{L i}\right)$ to guarantee power balance in the system while following operational 
goals. To do so, the power from the MS and the battery should equal the required power by the connected critical and non-critical loads, as shown below.

$$
\begin{gathered}
P_{\text {in }}(k)-P_{\text {batt }}(k)-\sum_{i} S_{L i}(k) P_{L i}^{\text {shed }}(k)-\sum_{j} P_{L j}^{\text {nonshed }}(k)=0 \\
P_{\text {batt }}(k)=P_{\text {ch }}(k)-P_{\text {disch }}(k)
\end{gathered}
$$

$\mathrm{Eq}(2)$ shows the battery power being equal to $P \operatorname{ch}(k)$ and $P$ disch $(k)$ during charging and discharging time, respectively. The charging/discharging power of the battery is the control command that needs to be determined by the EPS control system by directly adjusting the $P_{\text {in }}$ as load power changes. The SOC of the battery as the system state variable is evaluated using the discrete dynamic equation in (3), which is essential for predicting the future status of the ESS.

$$
\operatorname{SOC}(k+1)=\operatorname{SOC}(k)+T_{s} \cdot \eta_{c h} \cdot \frac{P_{c h}(k)}{B_{c a p}}-T_{s} \cdot \frac{P_{\text {disch }}(k)}{\eta_{\text {disch }} \cdot B_{\text {cap }}}
$$

According to (1)-(3), the system model includes both continuous variables related to the power from the MS and the ESS as well as binary variables representing the connection/disconnection status of the noncritical loads. Besides, to avoid simultaneous charging/discharging of the battery, additional binary variables are introduced to show the charge/discharge status of the battery at each stage. The system dynamics introduced above together with operation limitations over the prediction horizon are considered as constraints in a MPC-based controller, which is presented in detail in Section IV. The core of the MPC strategy is a model of the system under control to predict the evolution of system output over the desired horizon. Thus, the system model introduced in this section is repeatedly updated based on the most recent information obtained from the system. Besides, a prediction of the system load over the prediction horizon is required.

The MEA load can potentially be predicted by online machine learning-based methods combining the adjustment of the flight stages, height, and historical data [24], [39], which is out of the scope of this study. In this paper, the sampled load profile is used for prediction without adjustment for real-time flight power changes, and the deviations between the predicted load and the real values are neglected to simplify the analysis.

\section{MILP-MPC Controller Framework}

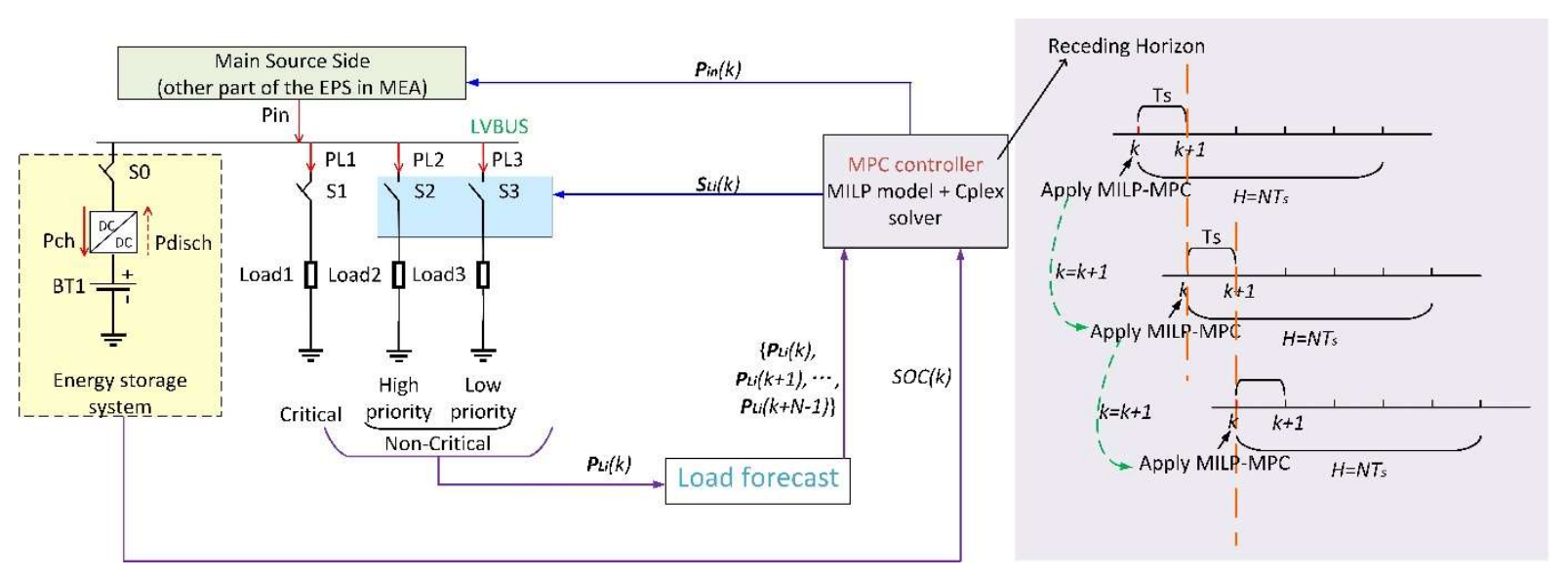

Figure 2. Online MILP-MPC optimization procedure with receding horizon

The proposed MPC-based control architecture is represented in Figure 2. This paper concentrates on the system-level MPC controller, which is assumed to be weakly dependent on the transient behavior of the fast control dynamics. As can be seen in Figure 2, the 
controller received the updated information of the system at the beginning of each control cycle defined by the desired sampling time. This information includes the updated network constraints, battery status, and the prediction of the load demands and associated priorities.

The control problem of the target MEA is formulated as a dynamic MILP optimization problem and the CPLEX solver [40] is used to solve the problem at each time step, guaranteeing to find an optimal solution for each time step. The MS-ES-L system is modeled as a discrete-time dynamic system with sampling time $T_{s}$ as introduced in the previous section. The MILP-MPC controller framework is structured as follows.

\subsection{MILP-MPC framework}

As illustrated in Figure 2, at each time step $k, k=0,1, \ldots, N-1$, an online optimization problem is solved over the finite prediction horizon $H$. The prediction horizon indicates the time looked ahead in the future to evaluate the cost function subject to several technical and operational constraints, which can be calculated as $H=N T_{s}$ [41]. The MPC works based on a receding horizon scheme presented in Figure 2. At each time step $k$, the MPC controller receives the updated status of the system and finds the solution of a finite horizon optimization problem. The result is an optimal control sequence over the optimization horizon. However, only the first sample of the optimal control sequence is applied to the system, and the control problem is shifted to the next time step $k+1$ as shown in Figure 2. The MPC control procedure is explained in detail in the following. The controller aims to keep the battery energy storage highly charged, with fewer power variations to prolong the battery life cycle, and to minimize load shedding, with less contactor switching which needs to be appropriately modeled in the MPC optimization problem. The optimization problem including these operating criteria and system constraints is introduced in the next section.

\section{MILP-MPC controller}

1) Update system state at time step $k$

a) Initialize the model with the measured/estimated state of the components, e.g. $\operatorname{SOC}(k)$, each load power measurement $\boldsymbol{P}_{L i}(k)$

b) Predict load demands over the prediction horizon $H$ at each future sampling time $T_{s,}$ e.g. $\boldsymbol{P}_{L i}(k), \boldsymbol{P}_{L i}(k+1), \ldots, \boldsymbol{P}_{L i}(k+N-1)$

2) Perform optimization: the high-level controller computes the optimal input sequence for the prediction horizon $H$, based on the updated system status and the predictions of the future system behavior. The optimal input sequence includes the decision of load shedding or not $\left(S_{L i}, k=0,1, \ldots, N-1, H=N T_{s}\right)$ and the input power reference on the MS-side $P_{\text {in }}\left(k=0,1, \ldots, N, H=N T_{s}\right)$. The optimization problem is solved using the CPLEX solver.

3) Apply the first sample of the optimal control sequence.

4) Set $k=k+1$, go to step 1 , and repeat the process with the new state.

\section{Formulation of Objectives and Constraints in MPC}

The MEA system control problem can be mathematically modeled using a set of technical and operational constraints and objective functions. In this section, three different objective functions are proposed for multiple control targets, and the system constraints are introduced.

\subsection{Designing the objective functions}

There are several control targets followed by the MEA system under study. Firstly, the controller needs to minimize the total time for which loads are shed while maximizing the time duration for which the energy stored in the battery is kept within the target range $(\mathrm{SOC}(k) \in[\mathrm{LO}, \mathrm{HI}])$. Besides, it needs to be ensured that the high priority loads are less 
shed than the low priority loads. Regarding the load management, instead of shedding/connecting loads continually, it is preferable to keep the contactors' switching activity as low as possible to prolong the components lifetime, and to avoid the transients and instabilities in the system, even though this may potentially increase the total time for which loads are disconnected. Moreover, the battery is preferred to be charged/discharged smoothly rather than constantly changing the status between charging and discharging modes to improve the life span of the battery [42]. Apart from load shedding targets, the battery management-related goals could be formulated with different cost functions as well as different weighting factors for battery charging/discharging power, maintaining battery SOC, and minimizing battery power variation. The following three objective functions are therefore proposed to formulate the desired operating criteria and to analyze the effects of different cost functions on the system performance.

4.1.1 The first proposed objective function (Obj1)

Obj1 in (4) denotes the first objective function combining the cost functions in (5)(9) for each target by different weighting factors $w_{S}, w_{\delta}, w_{P c h}, w_{P d i s c h}$, and $w_{\Delta}$. Cost function (5) indicates minimizing the total load shedding time by penalizing the shedding of loads with the load priorities. The change in load connections (connected/disconnected) for each time interval is minimized in (6). Since the energy stored in the battery can be calculated from the net power flowing to the battery, the cost function (7) and (8) can lead to maximizing the energy stored by penalizing the discharging and rewarding charging of the battery: the cost function in (7) encourages the system to charge the battery with as much power as is available, up to the rated value, while the cost function in (8) encourages the system to avoid battery discharging by minimizing the discharging power for every time interval. The charging speed of the battery can be changed by adopting different weighting factors for (7) and (8). For example, when $w_{P c h}>w_{S}$, the system will allow more load shedding to use power from the MS to charge the battery. When $w_{\text {Pdisch }} \gg w_{S} \gg w_{P c h}$, the system will shed loads when the battery is needed, but the charging will be slow as the MS priority is to supply loads rather than charging the battery.

$$
\mathrm{Obj} 1=w_{S} J_{S_{L i}}+w_{\delta} J_{\delta_{L i}}+w_{P c h} J_{P_{c h}}+w_{P d i s c h} J_{P_{\text {disch }}}+w_{\Delta} J_{\Delta}
$$

Where

$$
\begin{gathered}
J_{S_{L i}}=\sum_{k=0}^{N-1} \sum_{i=1}^{N_{L i}} \gamma_{L i}\left(1-S_{L i}(k)\right) / N \sum_{i=1}^{N_{L i}} \gamma_{L i} \\
J_{\delta_{L i}}=\sum_{k=0}^{N-1} \sum_{i=1}^{N_{L i}}\left|S_{L i}(k+1)-S_{L i}(k)\right| / N \cdot N_{L i} \\
J_{P_{c h}}=\sum_{k=0}^{N-1}\left(P_{c h}^{\max }-P_{c h}(k)\right) / N P_{c h}^{\max } \\
J_{P_{\text {disch }}}=\sum_{k=0}^{N-1}\left(P_{\text {disch }}(k)\right) / N P_{\text {disch }}^{\max } \\
J_{\Delta}=\sum_{k=0}^{N-1}(\varepsilon(k)+\vartheta(k)) / N
\end{gathered}
$$

For battery management, the battery SOC is preferred to be kept in a target range $[\mathrm{LO}, \mathrm{HI}]$ that can be modeled as a constraint. However, when there is a deviation between the predicted load and the realized load, the battery SOC might be temporarily out of the target range, which would prevent the solver from finding a feasible solution. Hence, rather than enforcing this requirement as a hard constraint, two additional variables $(\varepsilon(k)$ and $\vartheta(k)$ ) are introduced to penalize the deviation of the battery SOC from the the target lower and upper bounds in the objective function as presented in (9). In this way, it is tried 
to keep the SOC within the target range or minimize the deviations. These variables are explained further in Subsection 4.2 below.

\subsubsection{The second proposed objective function (Obj2)}

Adding the cumulative battery SOC to the objective function will increase the average energy stored in the battery. $\mathrm{Eq}(8)$ represents a normalized cost function to increase the battery SOC to be as close as possible to the upper bound. Hence, Obj2 in (10) is formed by adding the battery SOC cost function to Obj1 in (4), with its weighting factor $w_{S O C}$. Compared to the cost for charging/discharging power in (7) and (8), including SOC in the cost function will directly control SOC to maintain the desired reference value, which can be adjusted according to the applications. In this work, the desired reference value is set to be HI. Moreover, the charging will speed up more when longer prediction horizons are selected, as the SOC is a time-accumulated value, reflecting the accumulated charge for the whole prediction horizon.

$$
\text { Obj2 }=w_{\delta} J_{\delta_{L i}}+w_{S} J_{S_{L i}}+w_{S O C} J_{S O C}+w_{P c h} J_{P_{c h}}+w_{P d i s c h} J_{P_{\text {disch }}}+w_{\Delta} J_{\Delta}
$$

Where

$$
J_{S O C}=\sum_{k=0}^{N-1}|H I-S O C(k+1)| /(N \cdot H I)
$$

\subsubsection{The third proposed objective function (Obj3)}

The aforementioned objective functions control the energy stored in the battery. However, proposing a cost function to prolong the battery life is also important for aircraft EPS for longer-term safe flights, for example, reducing the battery charging/discharging current variations and reducing charging/discharging operations [18]. In this work, it is assumed that the battery voltage has small deviations, so that reducing the battery charging/discharging current variations is equivalent to reducing the charging/discharging power variations. This also reduces the charging/discharging operations to reduce the charging cycle, which also benefits the battery lifetime. The cost for minimizing the battery charging/discharging power change $J_{P_{\text {batt }}}$ is presented in (13), where $P_{b a t t}(k)=P_{c h}(k)-P_{\text {disch }}(k)$ presents the battery net power. Obj3 represented in (12) is formulated by adding this prolonging battery lifetime cost to Obj2 in (10) with the weighting factor $w_{\text {Pbatt }}$.

$$
\mathrm{Obj3}=w_{\delta} J_{\delta_{L i}}+w_{S} J_{S_{L i}}+w_{S O C} J_{S O C}+w_{P c h} J_{P_{c h}}+w_{\text {Pdisch }} J_{P_{\text {disch }}}+w_{\text {Pbatt }} J_{P_{\text {batt }}}+w_{\Delta} J_{\Delta}
$$

Where

$$
J_{P_{\text {batt }}}=\sum_{k=0}^{N-1}\left|P_{b a t t}(k+1)-P_{b a t t}(k)\right| / N\left(P_{c h}^{\max }+P_{\text {disch }}^{\max }\right)
$$

\subsection{System constraints}

1) Power balance constraints

For the LV bus, the sum of power flowing into/out of it equals zero (Kirchhoff's Current Law for a given voltage), assuming no losses within the bus itself. Hence, for the system presented in Figure 1, the power from the MS and the battery equals that required by the connected critical and non-critical loads, which can be formulated as (1) and (2), which is mentioned in Section 2.

\section{2) Storage dynamics}

The battery SOC can be calculated from the charging/discharging power over time using (3) presented in Section 2 [32].

3) Hard and soft constraints of SOC 
Battery $S O C \in[0,1]$, where $S O C=1$ indicates a fully charged battery, while $S O C=0$ corresponds to a depleted battery. In the aircraft, $S O C$ is preferred to be kept within a target range [LO, HI]. Therefore, upper and lower bounds are defined in (14), where $\mathrm{LO}=0.3, \mathrm{HI}=0.9$ is selected in this case, which could be presented as hard constraints for the optimization.

$$
0.3 \leq \operatorname{SOC}(k) \leq 0.9
$$

However, the hard constraints (14) increase the sensitivity of the algorithm to the power fluctuations. Because SOC is related to the stored or released energy, which is influenced by the power fluctuations, it is necessary to allow SOC to vary slightly outside of this range in real situations, due to unpredicted power consumption variations. In this case, the hard constraints (14) would prevent finding a feasible solution. Therefore, soft constraints in (15) - (17) are proposed in our model to improve the robustness of the MILPMPC algorithm. Variables $\varepsilon(k)$ and $\vartheta(k)$ are introduced to relax the hard upper and lower bounds in (14). The maximum allowed deviation for both bounds is 0.1 in this case. By adding two large weight factors to $\varepsilon(k)$ and $\vartheta(k)$ in the cost function as presented in Obj1 (4) and (9), SOC will usually be kept within the target range [0.3, 0.9], while allowing some tiny deviations when necessary.

$$
\begin{gathered}
0.3-\varepsilon(k) \leq S O C(k) \leq 0.9+\vartheta(k) \\
0 \leq \varepsilon(k) \leq 0.1 \\
0 \leq \vartheta(k) \leq 0.1
\end{gathered}
$$

4) Charging/discharging mode constraints

The battery can be either charged or discharged by the system. Therefore, two binary indicators are introduced to represent different modes in (18), (19), and (20), allowing the modes to be treated differently.

$$
\begin{gathered}
\zeta_{c h}(k)+\zeta_{\text {disch }}(k)=1, \zeta_{c h}(k), \zeta_{\text {disch }}(k) \in\{0,1\} \\
0 \leq P_{c h}(k) \leq \zeta_{c h}(k) \cdot P_{c h}^{\max } \\
0 \leq P_{\text {disch }}(k) \leq \zeta_{\text {disch }}(k) \cdot P_{\text {disch }}^{\max }
\end{gathered}
$$

5) Bounds of input power from the MS-side

The input power from the MS-side is limited by the maximum power that can be transferred by the DC/DC converters, and these input power bounds are presented in (21)

$$
0 \leq P_{\text {in }}(k) \leq P_{\text {in }}^{\max }
$$

\section{Evaluation for Various Objectives}

The objective functions designed in Section IV lead to different control problems and consequently different operating strategies for the MS-ES-L system, which are also impacted by the prediction horizon and the load profile. To compare the differences caused by these factors, an evaluation framework is proposed in this section to quantify the control results for all flight stages. The overall flight time is $T=M \cdot T_{s}$, where $M$ is the total number of time intervals. Moreover, to verify that the proposed method is suitable for realtime applications, the computation time for the MILP-MPC controller should be less than the sample time $T_{s}$. As the computation time can be impacted by the objective function 
formulation, prediction horizon, and load samples, the impact of these factors on the computation time is also studied in this section.

\subsection{Model parameters}

The model parameters including the MS-ES-L system component parameters are listed in Table 2, and the weighting factors are listed in Table 3. These weighting factors are selected by the method proposed in [43]. The MILP-MPC controller sample time $T_{s}$ is selected as $1 \mathrm{~min}$, the verification for the sample time selection will be demonstrated in Subsection 5.3. The total flight time is $150 \mathrm{~min}$.

Table 2 Parameters for the MS-ES-L system

\begin{tabular}{c|c|c|c}
\hline \hline$B_{\text {cap }}$ & $4 \mathrm{kWh}$ & $P_{c h}^{\max }, P_{\text {disch }}^{\max }$ & $4 \mathrm{~kW}$ \\
\hline$P_{\text {in }}^{\max }$ & $4 \mathrm{~kW}$ & $\eta_{c h}, \eta_{\text {disch }}$ & $90 \%$ \\
\hline$P_{\text {Load }}^{\max }$ & $4 \mathrm{~kW}$ & $S O C(0)$ & 0.3 \\
\hline \hline
\end{tabular}

Table 3 Weighting factors in objective functions

\begin{tabular}{c|c|c|c}
\hline \hline$w_{S}$ & 5 & $w_{\delta}$ & 34.7 \\
\hline$w_{S O C}$ & 16 & $w_{\Delta}$ & $1 \mathrm{e} 5$ \\
\hline$w_{P b a t t}$ & 2 & $w_{P d i s c h}$ & 5.1 \\
\hline$w_{P c h}$ & \multicolumn{2}{|c}{3.1 in Obj1, 2.8 in Obj2 and Obj3 } \\
\hline \hline
\end{tabular}
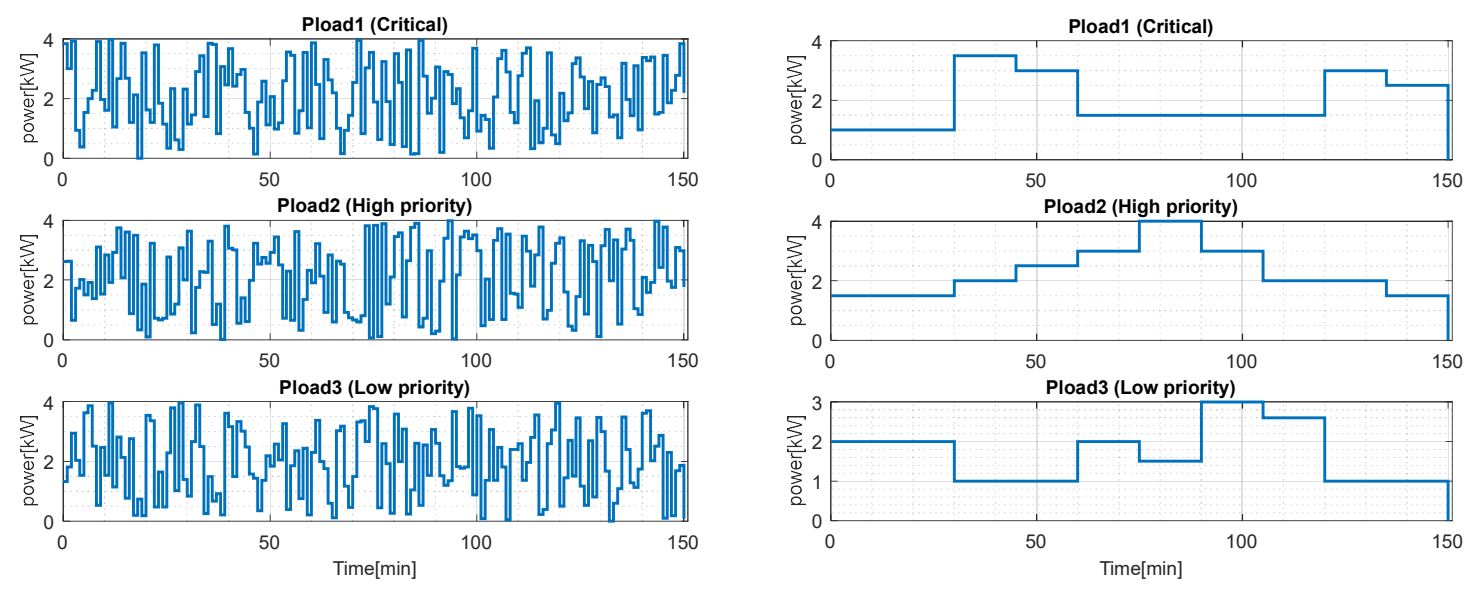

(a) Case 1: Random load profiles

(b) Case 2: Load profiles based on flight stages

Figure 3 Different load cases

For evaluating the load profile impacts on the results, two types of load profiles are used. In Figure 3 (a), a randomly changing load profile is considered, while Figure 3 (b) demonstrates the load profiles based on different flight stages. The flight stages include ground (pre-departure and taxiing-out), takeoff, climb, cruise, descent, landing, and taxiing-in. Each load system requires a different amount of power at each flight stage. For example, the cabin power demand reaches the highest level at the cruise stage which can be several times larger than the power demand at other stages. In addition, the power demand of the environment control system increases dramatically as soon as the aircraft takes off and keeps almost the same value for the following stages, while the landing gear only requires power during ground, takeoff, landing, and taxiing-in stages [44], [45]. 
Therefore, compared to the load profiles in Figure 3 (a) changing every minute, the profiles in Figure 3 (b) change much slower where each load level lasts at least for $15 \mathrm{~min}$. However, an average load power of $2 \mathrm{~kW}$ can be seen in both cases.

\subsection{Evaluation indices}

In the following subsections, eight evaluation indices are proposed. The control results are evaluated by each of the proposed indices for both load scenarios when objectives Obj1-Obj3 are adopted for prediction horizons $H$ varying from $1 T_{s}$ to $30 T_{s}(\mathrm{~N}=1,2, \ldots, 30)$. Each index represents the corresponding quantified performance, and lower values indicate better performance.

\subsubsection{Load Shedding index}

The normalized load shedding index quantifies the ratio of shed loads throughout the whole flight time $T=150 \mathrm{~min}$, as shown in (22).

$$
G_{S_{L i}}=\sum_{k=0}^{M-1} \sum_{i=1}^{N_{L i}}\left[\gamma_{L i}\left(1-S_{L i}(k)\right)\right] /\left(M \cdot \sum_{i=1}^{N_{L i}}\left(\gamma_{L i}\right)\right)
$$

In Figure 4, the load shedding index values are compared when adopting different prediction horizons and objective functions, with case 1 and case 2 showing the results for adopting different load profiles. For both load cases, the Obj2-3 have similar changes: when the prediction horizon is short, load shedding remains lower than for Obj1, while the load shedding increases quickly as the prediction horizon becomes longer, causing Obj2-3 to have more load shedding than Obj1 with long prediction horizons. Although the load shedding for Obj1 also has the incrementally increasing tendency when $N$ increases for the second load case, the growth rate is much slower compared to Obj2-3. This difference between Obj1 and Obj2-3 is caused by the cost function Jsoc - more loads are shed to speed up the SOC accumulating when $N$ increases.

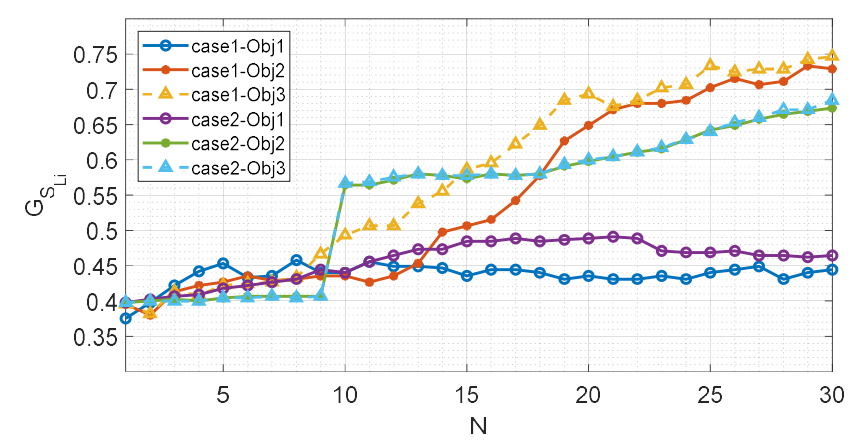

Figure 4 Comparison of different prediction horizons and objective functions based on the normalized shedding index for two load cases

Comparing Obj2 and Obj3, when the loads frequently vary, as presented in case 1, the load shedding for Obj3 increases at a higher rate than Obj2, while Obj3 has less load shedding than Obj2 when $N \leq 6$. This is caused by the cost function JPbatt in Obj3. By changing the load shedding scheduling and adjusting the input power from MS, the battery can be charged/discharged smoothly. Combining with Jsoc, more load shedding will be conducted as $N$ increases so that the system keeps charging the battery with fewer power fluctuations. When the load varies less and slowly as load profiles in case 2, the load shedding curves for Obj2 and Obj3 are mostly overlapped, because the stable load profile lacks the scheduling possibilities while the WF for JPbatt is small, hence Jsoc dominates JPbatt in this case. Comparing the results for $N=9$ in Figure 4, there is a sharp rise of the index value at $N=10$, which indicates that the shedding of high-priority loads is increased.

In summary, Obj1 has better performance in load shedding compared to Obj2-3 on average. However, when the selected prediction horizon is not too long, e.g. $N \leq 9$ in our case, the Obj2-3 can have better load shedding performance. 


\subsubsection{Contactor status change index}

The average number of connection status changes for each contactor in the whole time period is presented in (23), where $N_{L i}$ indicates the total number of non-critical shreddable loads.

$$
G_{\delta_{L i}}=\sum_{k=0}^{M-1} \sum_{i=1}^{N_{L i}}\left|S_{L i}(k+1)-S_{L i}(k)\right| /\left(M \cdot N_{L i}\right)
$$

These index values are compared in Figure 5 when adopting different prediction horizons and objective functions, with case 1 and case 2 showing the results for different load profiles. In general, the change of contactor status is reduced as $N$ increases in both cases for Obj1-Obj3, while the curve for Obj1 in case 2 fluctuates the most. In both load cases, the contactor change values for Obj2-3 decrease very quickly when $N<10$ and then keep a low level when the prediction horizon is longer. Moreover, when $N$ is in this range, compared with the results for load shedding in Figure 4, Obj2-3 also has good performance for load shedding index. This also benefits from the inclusion of $J s o c$, as it takes results of power integration over time into consideration within the prediction horizon.

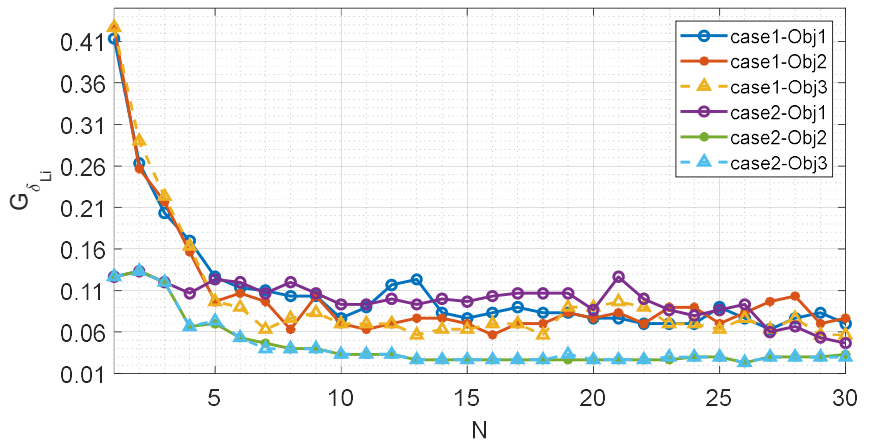

Figure 5 Comparison of different prediction horizons and objective functions based on the contactor status change index for two load cases

It can be concluded that Obj2 and 3 perform better than Obj1 for maintaining the contactor status in different load cases. Moreover, for the contactor status change index, a very long prediction horizon is unnecessary for Obj2-3, saving computation time while still achieving good results.

\subsubsection{Battery energy storage level index}

In aircraft applications, it is preferable to keep the battery highly charged to cope with emergency cases and unforeseen situations. Hence, when designing the optimization algorithms, the objective functions are designed to maximize the average energy stored in the battery. The index (24) is proposed to evaluate the average level of the energy stored during the studied scenarios.

$$
G_{S O C}=\sum_{k=0}^{M-1}|0.9-S O C(k)| /(M \cdot 0.9)
$$

Figure 6 shows the comparison of the Gsoc index values with different prediction horizons and objective functions, with case 1 and case 2 showing the results for adopting different load profiles. In general, the energy storage level index value is reduced as $N$ increases in both cases for Obj1-Obj3, while for the load case 1, the index values for MILP $(N=1)$ are less than the ones for MILP-MPC $(N \geq 2)$ when $N \leq 12$ for Obj3, $N \leq 16$ for Obj2, and $N \leq 30$ for Obj1. When $N=1$, the contactor status changes will not impact the optimization results for each time step. Combined with the frequently varying loads, the SOC is therefore improved by shedding/connecting loads with high power demands continuously. However, when $N=2$, the number of contactor status changes is much reduced (the index value is reduced by 1.3) compared to the value when $N=1$. In the meantime, the battery 
will be used for supporting loads even the loads' demands could be high, which slows down the SOC accumulation.

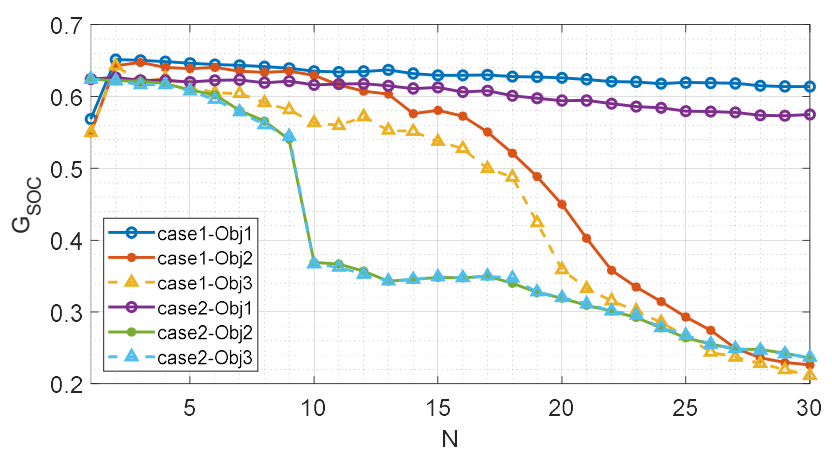

Figure 6 Comparison of different prediction horizons and objective functions based on the battery energy storage level index for two load cases

Comparing the SOC changing rate for Obj1-3 with the MILP-MPC method, for both load cases, Obj2 and 3 improve SOC level at a higher rate than Obj1 when $\mathrm{N}$ increases, since both Obj2 and Obj3 benefit from the cost function Jsoc to speed up the energy storage in the long term. For load case 1, Obj3 speeds up the SOC accumulation with a higher rate than Obj2, as the cost function JPbatt in Obj3 tends to keep the battery charging with few power fluctuations.

This suggests that Obj3 has the best performance for the battery storage level index. When adopting Obj3, the prediction horizon does not necessarily need to be selected too long, as it charges the battery much faster than Obj1.

\subsubsection{Battery power change index}

The battery life will be shortened when the battery power is frequently changed [18]. The index (25) is therefore proposed to calculate the total battery power change for all flight stages.

$$
G_{P_{\text {batt }}}=\sum_{k=0}^{M-1}\left|P_{\text {batt }}(k+1)-P_{\text {batt }}(k)\right| / M\left(P_{c h}^{\max }+P_{\text {disch }}^{\max }\right)
$$

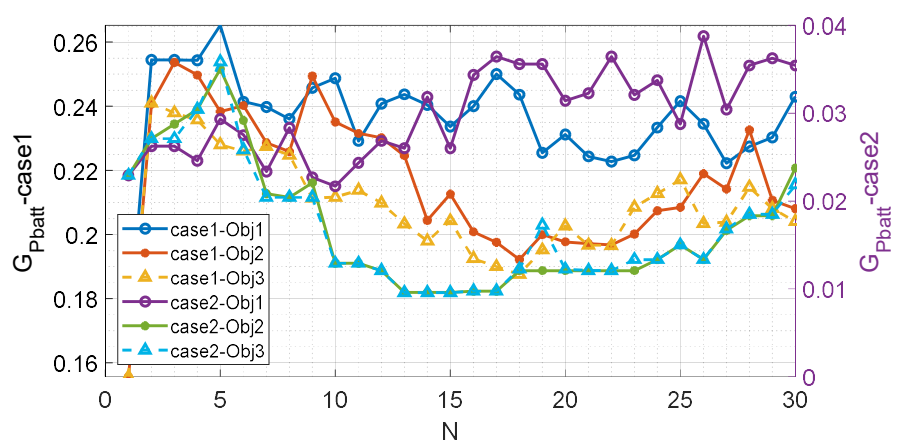

Figure 7 Comparison of different prediction horizons and objective functions based on the battery power change index for two load cases

Figure 7 presents the comparison of the battery power change index values with different prediction horizons and objective functions, with case 1 and case 2 showing the results for adopting different load profiles. In both load cases, for each $N$, Obj3 usually has a better performance compared to Obj1, because Obj1 doesn't contain the cost function $J$ Pbatt to minimize the power change. However, as Obj2 has the cost function Jsoc in common with Obj3 with relatively high WF, the index value curve of Obj2 has similar trends as Obj3 - the value is reduced to the minimum value and then slightly rises with variations 
when $N$ increases from 2 to 30 . Because the load shedding and the reduction of the load on/off changes can avoid the battery changing between the charging and discharging modes, Obj2 and Obj3 perform similarly in these indices. By adding the cost function JPbatt in Obj3, the charging power can be smoothed whenever it is possible. On the other hand, when $N$ increases $(N>20)$ to keep accumulating SOC, more power will be injected into the battery, thereby increasing the battery charging power variation. Moreover, JPbatt and Jsoc can impact the results for load shedding and the contactor status changes, which results in the battery power change fluctuations, as can be seen in Figure 7.

Based on this comparison, Obj3 has the best performance for the index of battery power change, which also indicates that this objective function is preferred when battery life is important.

\subsubsection{SOC target range index}

Since the soft constraints of SOC are introduced to improve the robustness of the algorithm, the real SOC might exceed the target range of [0.3, 0.9]. The SOC target range index is built to evaluate the ratio of the outrange SOC by which the SOC is exceeded. Equations (26) and (27) are considered for the upper and lower bounds respectively, where $S$ is the set of all time intervals which satisfy the desired ranges. The SOC can be out of range in the following situations: 1) there are deviations between the predicted and realized load which the controller cannot handle in time; 2 ) the controller has a delay in sending and receiving signals. In this section, the ideal situation is considered, hence the SOC target range index remains 0 .

$$
\begin{gathered}
G_{\overline{S O C R}}=\sum_{k \in S:(\operatorname{SOC}(k)>0.9)}(\operatorname{SOC}(k)-0.9) /(1-0.9) \\
G_{\underline{\underline{S O C R}}}=\sum_{k \in S:(\operatorname{SOC}(k)<0.3)}(0.3-\operatorname{SOC}(k)) / 0.3
\end{gathered}
$$

\subsubsection{Index for multi-objective problem}

Although the performance of each objective function has been analyzed in 1) - 5) for each index, the overall performance of each objective function concerning the length of prediction horizon $N$ still needs to be investigated. For a multi-objective problem with conflicting cost functions, e.g. Jsoc and JSLi, there are trade-offs among the evaluation indices. Hence, weight parameters for each index are selected to represent the practical requirements, i.e. priorities of each optimization target. The final overall index is formed with the full knowledge of system performance throughout the whole operation, by using the above-mentioned indices, which are combined with appropriate user-specified weight parameters. The WFs utilized by the MPC to get the best overall results may differ from the weights used in the final evaluation. A neural network-based method in [43] is adopted for selecting WFs and weights in the overall index. The multi-objective evaluation index is presented in (28) with the adopted weights listed in Table 4.

$$
G_{M O}=v_{S} G_{S_{L i}}+v_{\delta} G_{\delta_{L i}}+v_{S O C} G_{S O C}+v_{P b a t t} G_{P_{\text {batt }}}+v_{\overline{S O C R}} G_{\overline{S O C R}}+v_{\underline{S O C R}} G_{S O C R}
$$

Table 4 Weights for the multi-objective index

\begin{tabular}{c|c|c|c}
\hline \hline$v_{S}$ & 2.8 & $v_{\delta}$ & 1.68 \\
\hline$v_{S O C}$ & 1 & $v_{\text {Pbatt }}$ & 0.4 \\
\hline$v \overline{\text { SOCR }}$ & $10^{4}$ & $v_{\text {SOCR }}$ & 104 \\
\hline \hline
\end{tabular}

Figure 8 presents the comparison of the proposed multi-objective evaluation index values with different prediction horizons and objective functions, as case 1 and case 2 show the results for adopting different load profiles. The figure shows that Obj2 and 3 can provide the optimal solutions with a shorter prediction horizon compared with Obj1. Compared to Obj2, Obj3 reaches a lower index value with a shorter horizon for load case 
1. These results are heavily based on the selection of the weights for each index, but with the adjustment of the weights by the users according to their application requirements, the optimal objective function with a suitable prediction horizon can be selected.

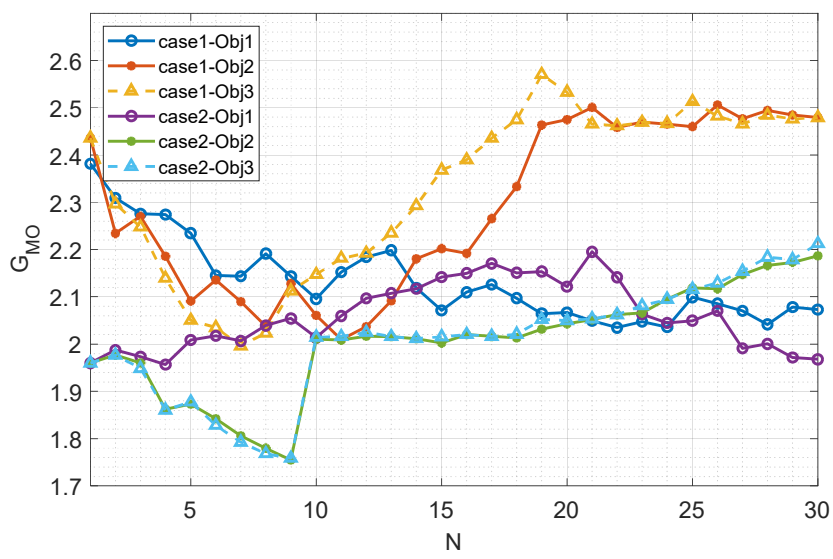

Figure 8 Comparison of different prediction horizons and objective functions based on the multiobjective index for two load cases

\subsection{Real-time application analysis}

For real-time MPC applications, computation time is the key issue to be considered, as the time required to complete the computation should be less than the sampling time. When adopting the CPLEX solver, the computation time is influenced by several factors: 1) more complex models will often take longer time; 2 ) longer prediction horizons will take longer time; 3) different input values, such as different load data as inputs to the MPC controller, can cause different computation times. Hence, to verify the MPC real-time application, the worst-case analysis of the computation time is conducted for Obj1 - 3. For each objective function and each N, the MPC controller will finally adopt 150 optimal solutions (one sample at each time step), and the maximum computation time for the MPC controller is recorded as the worst-case.

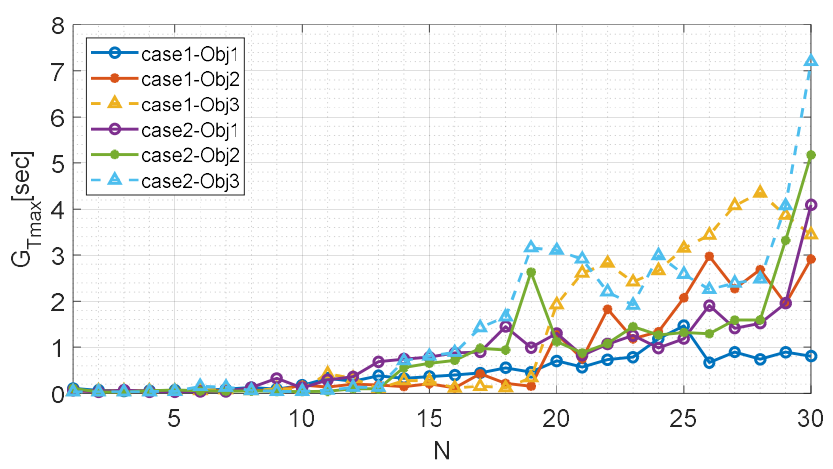

Figure 9 Comparison of the worst-case computation time of different prediction horizons and objective functions for two load cases

Figure 9 presents the maximum computation time $G_{T \max }$ changes for each objective function with respect to $N$. In both load cases, the maximum computation time for Obj1 3 has similar incrementally increasing tendencies as $N$ increases: when $N \leq 10$, the computation time is less than $0.2 \mathrm{~s}$; when $10<N \leq 15$, the computation time is less than $1 \mathrm{~s}$; when $15<N$, the computation time will go above $1 \mathrm{~s}$ with the maximum value less than $8 \mathrm{~s}$. This indicates that the proposed MPC method could be adopted for real-time application with selected sample times $T_{s}$ larger than 10s, during which the optimization would be completed, with the additional time being used for data measuring and communicating [46]. 
It is worth noting that a maximum computation time can be set in CPLEX. In this case, if the solver ran out of time, it would return the best solution that has been found in that time, rather than the optimal solution. In our case, $T_{s}$ is selected to $1 \mathrm{~min}$ because of the following reasons:

1) A 1-min sample time can provide the controller with enough time to obtain the measured status and updated load prediction, complete the optimization, and send the control signals to the system.

2) The proposed MPC method is mainly designed for satisfying the long-term battery SOC and load management requirements in the high-level control system. Since the SOC and average loads will not change very much in a few seconds, the MPC controller would not be required to update the control references frequently in small time intervals in the order of seconds. On the other hand, if the MPC is required to run every few seconds, to update the reference to cope with transients, the battery power and loads might change with every load fluctuation, which might cause instability in the system. In fact, instead of using MPC with long-term operating goals to act fast in response to the transient load changes, another real-time controller is usually added to the system at a lower control level, which will respond to the transient issues. This is the future work of the authors that will be presented in the future work discussion.

In summary, when adopting the proposed multi-objective evaluation index as the index for our overall preferred performance, Obj3 will have the best performance with a suitable prediction horizon ( $N=7$ and $N=9$ for the corresponding load cases), with a computation time of lower than $0.2 \mathrm{~s}$.

\section{Experimental Verification}

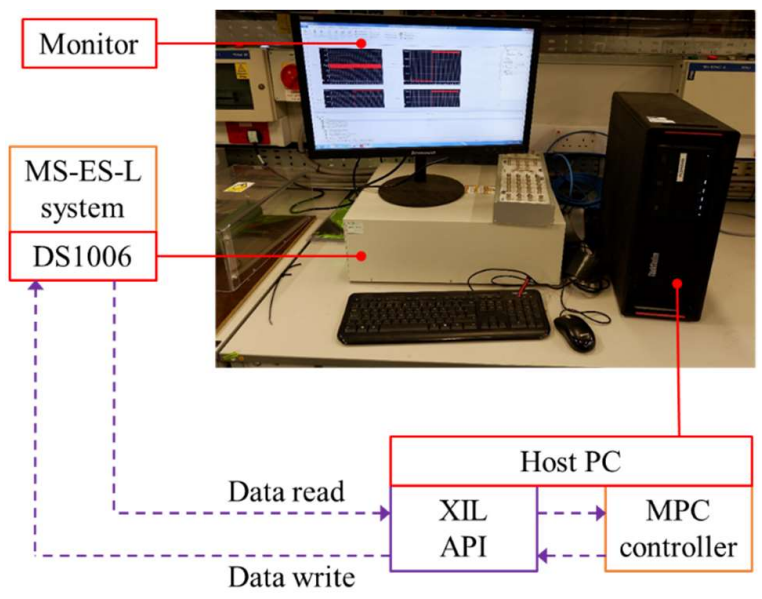

Figure 10 Experimental setup

A HIL experiment is implemented in the lab at the Aerospace Technology Centre at the University of Nottingham, as presented in Figure 10. The MS-ES-L system is implemented in dSPACE 1006 for real-time simulation. The MILP-MPC controller is run in Matlab on a host PC, connected with the dSPACE. Every $T_{s}$, Matlab on the host PC uses the XIL API interface to read the measured data from dSPACE, including the load power $P_{L i}(k)$ and battery SOC $(k)$. The MILP-MPC controller in Matlab takes these values as inputs and calls the CPLEX solver to provide the optimal power reference on the MS side $P_{i n}(k)$ and load shedding $S_{L i}(k)$. Finally, these optimal values are sent to the system implemented in ASPACE by the XIL API interface to realize real-time control. It is worth mentioning that the main purpose of this experiment is validating the suitability of the proposed system-level control framework for real-time implementation and the capability of the controller to provide the system with optimal control commands at specified control intervals 
to achieve the desired operating performance. However, deploying hardware devices representing the MS-ES-L system component as well as including device-level control systems will help evaluate the performance of the proposed system-level strategy in the full control hierarchy which is the scope of future work of the authors.

The control results of MPC with Obj1 - Obj3 for all flight stages are compared in this experiment. During the flight, the loads are changed following the profile in Figure $3(b)$. According to the analysis in Section V, the MPC for Obj3 performs best when $N=9$ with a computation time of less than $0.2 \mathrm{~s}$. To verify this analytic conclusion based on the mathematical model, the MPC with Obj1 -3 when $N=9$ is applied in the experiment. The performance of each objective function can be visualized by observing the figures presenting the SOC changes, load shedding results, and battery power change. The real-time application of MPC is also verified. It should be noted that the flight time slot has been scaled down from $60 \mathrm{~s}$ to $3 \mathrm{~s}$ to experiment within $7.5 \mathrm{~min}$ instead of $150 \mathrm{~min}$. The battery capacity is accordingly scaled down to $0.2 \mathrm{kWh}$ during the experiment. From both analyses in Figure 8 and the experiment, $\mathrm{Obj} 2$ and $\mathrm{Obj} 3$ have the same control results when $N=9$ for this load profile. Hence, in the following comparisons, only the control results for Obj1 and Obj3 are considered, to study the differences.

Figure 11 presents the SOC measurement results during the flight stages, while (a) shows the results when MPC adopts Obj1 and (b) is related to Obj3. When Obj3 is adopted, the battery SOC is kept within $0.4-0.6$ for the most time intervals with a maximum value of 0.56 , while SOC is kept within $0.3-0.36$ for the most time intervals when adopting Obj1, with a maximum value of 0.46 . As the SOC is close to the lower limit when adopting Obj1, the system will be less robust for coping with unexpected failure scenarios.

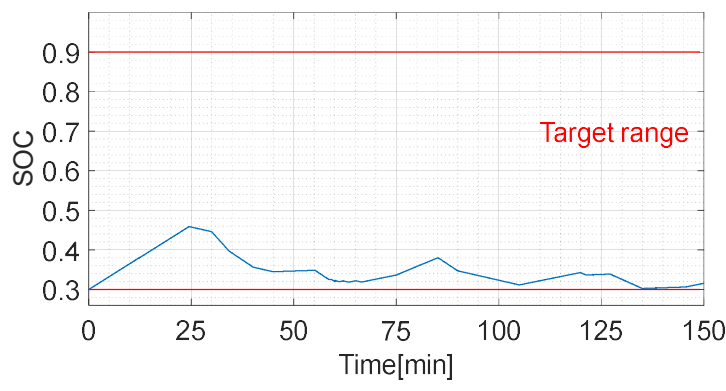

(a) SOC change with Obj1

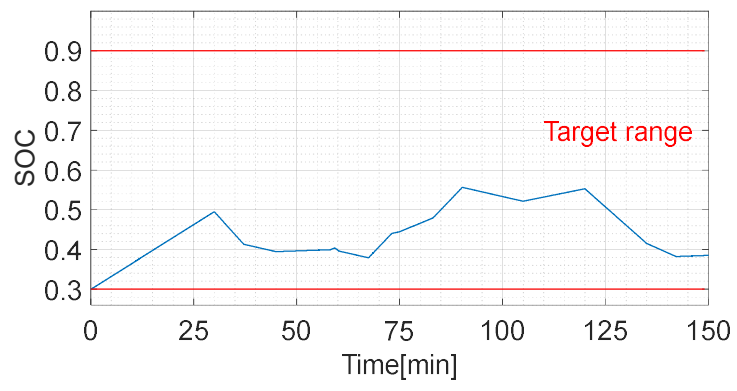

(b) SOC change with Obj3

Figure 11 SOC comparison for Obj1 and Obj3 when $N=9$
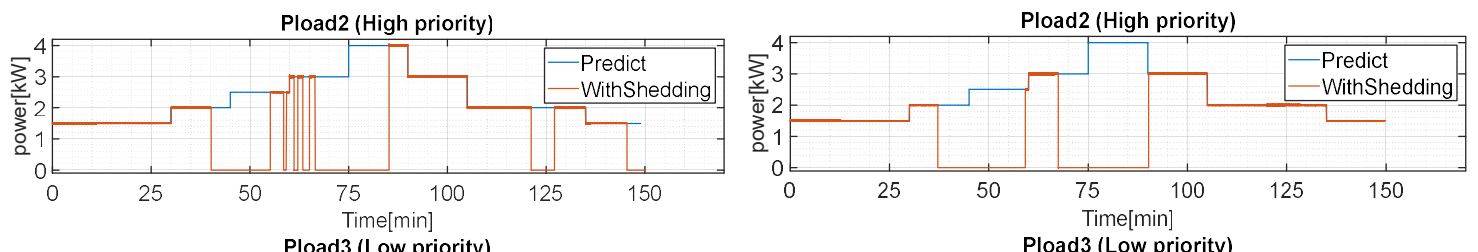

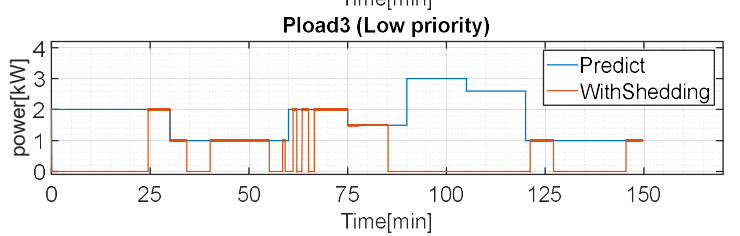

(a) Load shedding with Obj1

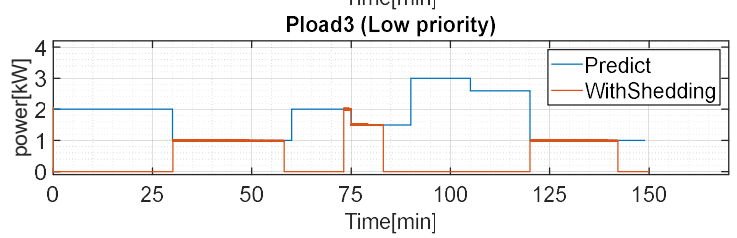

(b) Load shedding with Obj3

Figure 12 Load shedding comparison for Obj1 and Obj3 when $N=9$

The predicted and scheduled results for shedding of non-critical loads are presented in Figure 12, indicating load shedding in the system when adopting Obj1 in (a) and Obj3 
in (b). The load contactors have more frequent on/off activities when adopting Obj1 compared to the case of adopting Obj2. Also, when adopting Obj1, the minimum time period for keeping loads connected is $1 \mathrm{~min}$, while Obj3 will keep loads connected for at least 8 min, which is preferred for reducing transient issues. Although Obj3 has less load on/off activities, it still has less load shedding time for both high and low priority loads: the load shedding index for Load 2 is 0.317 with Obj1 compared to 0.299 with Obj3, while the load shedding index value for Load 3 is 0.617 with Obj1 compared to 0.599 with Obj3. In conclusion, Obj3 outperforms Obj1 in both load shedding and switching activities in the experiment.

The battery charging/discharging power measurements are presented in Figure 13, where (a) presents the results with Obj1, while (b) presents the results with Obj3. Comparing the two figures, when adopting Obj3, the battery has less switching between the charging and discharging modes, and the charging/discharging power is less changed during each mode. In addition, the battery power curve with Obj1 has several transient peaks, which happens when Load2 is connected/disconnected simultaneously with the Load3 connection action being reversed. This verifies that Obj3 performs better in terms of battery lifecycle as well as system stability.

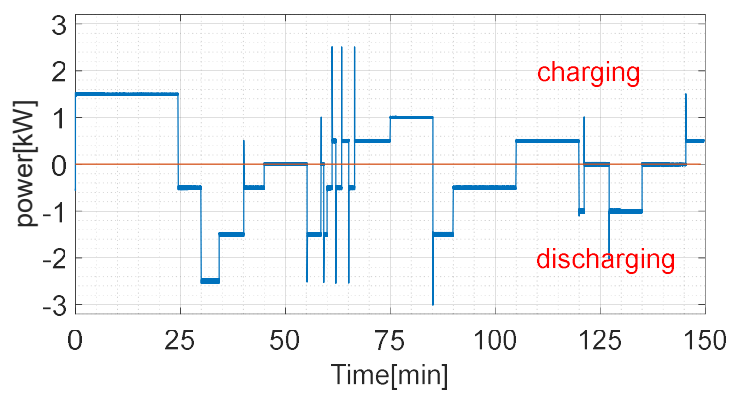

(a) Battery power with Obj1

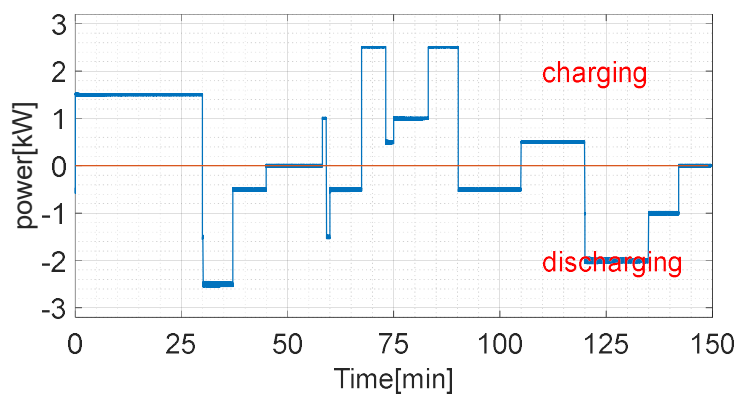

(b) Battery power with Obj3

Figure 13 Battery power comparison for Obj1 and Obj3 when $N=9$

In summary, the MPC model with Obj1 - 3 proposed in Sections III and IV is applicable for real-time applications. Besides, it was found that the MPC with Obj3 performs better than Obj1 in the real-time HIL experiment when $N$ is selected as 9 for the given load profile. This supports the analysis and conclusions in Section 5.

This research inspires our future work when the system extends to the whole complex MEA power system configuration, such as HV buses, APU, multiple bidirectional converters, etc. A hierarchical controller based on the MPC method is proposed to cope with more operation constraints and scenarios, where the idea and findings mentioned in this work are adopted to design the objective function. Moreover, the model proposed for optimization in MPC can be applied to the battery/EPS sizing problem, which helps to decrease the weight and volume of the aircraft. This topic will also be explored in our future research.

\section{Conclusions}

This paper proposed a system-level MPC framework to optimize the control and management of the energy storage and flexibility in the load-side for a MEA system. Following the control targets, three different objective functions were presented, and the importance of the different objective functions and prediction horizons was identified by analyzing the simulation results with the proposed evaluation framework including different performance indices. By assessing the impacts of different objectives, the paper provided the readers with a reference for selecting the most applicable objective function 
combination, as well as its potential impacts on deciding prediction horizons for different use cases.

\section{Acknowledgement}

This work is funded by the INNOVATIVE doctoral programme. The INNOVATIVE programme is partially funded by the Marie Curie Initial Training Networks (ITN) action, and partially by the Institute for Aerospace Technology (IAT) at the University of Nottingham. Najmeh Bazmohammadi and Josep M. Guerrero are with the Center for Research on Microgrids (CROM) supported by VILLUM FONDEN under the VILLUM Investigator Grant (no. 25920): www.crom.et.aau.dk

\section{References}

1. S. Bozhko, M. Liserre, K. Al-Haddad, G. Buticchi, and P. Wheeler, “On-board Microgrids for the More Electric Aircraft Technology Review," IEEE Trans. Ind. Electron., vol. 66, no. 7, pp. 1-1, 2018, doi: 10.1109/tie.2018.2881951.

2. D. Alfonso et al., "Batteries for Aerospace: a Brief Review," in 2018 AEIT International Annual Conference, 2018, vol. 217. doi: 10.23919/AEIT.2018.8577355.

3. C. Spagnolo, S. Sumsurooah, C. I. Hill, and S. Bozhko, "Finite state machine control for aircraft electrical distribution system," J. Eng., vol. 2018, no. 13, pp. 506-511, 2018, doi: 10.1049/joe.2018.0039.

4. C. Spagnolo, S. Sumsurooah, C. I. Hill, and S. Bozhko, "Smart controller design for safety operation ofthe MEA electrical distribution system," in IECON 2018 - 44th Annual Conference of the IEEE Industrial Electronics Society, vol. 1, pp. 5778-5785.

5. C. Spagnolo, S. Sumsurooah, and S. Bozkho, "Advanced smart grid power distribution system for More Electric Aircraft application," 2019 Int. Conf. Electrotech. Complexes Syst., pp. 1-6, 2020, doi: 10.1109/icoecs46375.2019.8949998.

6. A. Chaouachi, R. M. Kamel, R. Andoulsi, and K. Nagasaka, "Multiobjective intelligent energy management for a microgrid," IEEE Trans. Ind. Electron., vol. 60, no. 4, pp. 1688-1699, 2013, doi: 10.1109/TIE.2012.2188873.

7. D. Arcos-Aviles, J. Pascual, L. Marroyo, P. Sanchis, and F. Guinjoan, “Fuzzy logic-based energy management system design for residential grid-connected microgrids," IEEE Trans. Smart Grid, vol. 9, no. 2, pp. 530-543, 2018, doi: 10.1109/TSG.2016.2555245.

8. H. Zhang, C. Saudemont, B. Robyns, and R. Meuret, "Comparison of different DC voltage supervision strategies in a local Power Distribution System of More Electric Aircraft," Math. Comput. Simul., vol. 81, no. 2, pp. 263-276, 2010, doi: 10.1016/j.matcom.2010.05.009.

9. S. Njoya Motapon, L. A. Dessaint, and K. Al-Haddad, “A comparative study of energy management schemes for a fuel-cell hybrid emergency power system of more-electric aircraft," IEEE Trans. Ind. Electron., vol. 61, no. 3, pp. 1320-1334, 2014, doi: 10.1109/TIE.2013.2257152.

10. Y. Zhang et al., "An Energy Efficient Power Management Solution for a Fault-tolerant More Electric Engine/Aircraft," IEEE Trans. Ind. Electron., vol. PP, no. c, pp. 1-1, 2018, doi: 10.1109/TIE.2018.2877169.

11. R. Francisco, C. Roncero-Clemente, R. Lopes, and J. F. Martins, "Intelligent energy storage management system for smart grid integration," Proc. IECON 2018 - 44th Annu. Conf. IEEE Ind. Electron. Soc., pp. 6083-6087, 2018, doi: 10.1109/IECON.2018.8591068.

12. C. C. Lin, D. J. Deng, C. C. Kuo, and Y. L. Liang, "Optimal Charging Control of Energy Storage and Electric Vehicle of an Individual in the Internet of Energy With Energy Trading," IEEE Trans. Ind. Informatics, vol. 14, no. 6, pp. 2570-2578, 2018, doi: 10.1109/TII.2017.2782845.

13. Y. Ma, P. Yang, Y. Wang, Z. Zhao, and Q. Zheng, “Optimal sizing and control strategy of islanded microgrid using PSO technique," Asia-Pacific Power Energy Eng. Conf. APPEEC, vol. 2015-March, no. March, 2014, doi: 10.1109/APPEEC.2014.7066004.

14. W. Al-Saedi, S. W. Lachowicz, D. Habibi, and O. Bass, "Power flow control in grid-connected microgrid operation using Particle Swarm Optimization under variable load conditions," Int. J. Electr. Power Energy Syst., vol. 49, no. 1, pp. 76-85, 2013, doi: 10.1016/j.ijepes.2012.12.017.

15. S. A. Pourmousavi, M. H. Nehrir, C. M. Colson, and C. Wang, "Real-time energy management of a stand-alone hybrid windmicroturbine energy system using particle swarm optimization," IEEE Trans. Sustain. Energy, vol. 1, no. 3, pp. 193-201, 2010, doi: 10.1109/TSTE.2010.2061881.

16. A. Soares, A. Gomes, C. H. Antunes, and C. Oliveira, “A customized evolutionary algorithm for multiobjective management of residential energy resources," IEEE Trans. Ind. Informatics, vol. 13, no. 2, pp. 492-501, 2017, doi: 10.1109/TII.2016.2628961.

17. T. Logenthiran, D. Srinivasan, and T. Z. Shun, "Demand side management in smart grid using heuristic optimization," IEEE Trans. Smart Grid, vol. 3, no. 3, pp. 1244-1252, 2012, doi: 10.1109/TSG.2012.2195686.

18. J. Shen and A. Khaligh, "A supervisory energy management control strategy in a battery/ultracapacitor hybrid energy storage system," IEEE Trans. Transp. Electrif., vol. 1, no. 3, pp. 223-231, 2015, doi: 10.1109/TTE.2015.2464690.

19. J. Moreno, M. E. Ortuzar, and J. W. Dixon, “Energy-management system for a hybrid electric vehicle, using ultracapacitors and neural networks," IEEE Trans. Ind. Electron., vol. 53, no. 2, pp. 614-623, 2006, doi: 10.1109/TIE.2006.870880. 
20. A. Parisio, E. Rikos, G. Tzamalis, and L. Glielmo, "Use of model predictive control for experimental microgrid optimization," Appl. Energy, vol. 115, pp. 37-46, 2014, doi: 10.1016/j.apenergy.2013.10.027.

21. A. Parisio, E. Rikos, and L. Glielmo, "A Model Predictive Control Approach to Microgrid Operation Optimization," IEEE Trans. Control Syst. Technol., vol. 22, no. 99, p. 1, 2014, doi: 10.1109/TCST.2013.2295737.

22. A. Parisio, C. Wiezorek, T. Kyntäjä, J. Elo, K. Strunz, and K. H. Johansson, “Cooperative MPC-Based Energy Management for Networked Microgrids," IEEE Trans. Smart Grid, vol. 8, no. 6, pp. 3066-3074, 2017, doi: 10.1109/TSG.2017.2726941.

23. A. C. Luna, J. M. Guerrero, J. C. Vasquez, M. Graells, and N. L. Diaz, “Mixed-Integer-Linear-Programming-Based Energy Management System for Hybrid PV-Wind-Battery Microgrids: Modeling, Design, and Experimental Verification," IEEE Trans. Power Electron., vol. 32, no. 4, pp. 2769-2783, 2016, doi: 10.1109/tpel.2016.2581021.

24. A. C. Luna, L. Meng, N. L. Diaz, M. Graells, J. C. Vasquez, and J. M. Guerrero, “Online Energy Management Systems for Microgrids: Experimental Validation and Assessment Framework," IEEE Trans. Power Electron., vol. 33, no. 3, pp. 2201-2215, 2018, doi: 10.1109/TPEL.2017.2700083.

25. N. Sockeel, M. Shahverdi, and M. Mazzola, "Impact of the State of Charge Estimation on Model Predictive Control Performance in a Plug-In Hybrid Electric Vehicle Accounting for Equivalent Fuel Consumption and Battery Capacity Fade," ITEC 2019 - 2019 IEEE Transp. Electrif. Conf. Expo, 2019, doi: 10.1109/ITEC.2019.8790581.

26. N. Sockeel, J. Shi, M. Shahverdi, and M. Mazzola, "Pareto Front Analysis of the Objective Function in Model Predictive Control Based Power Management System of a Plug-in Hybrid Electric Vehicle," 2018 IEEE Transp. Electrif. Conf. Expo, ITEC 2018, pp. 173-177, 2018, doi: 10.1109/ITEC.2018.8450164.

27. Z. Fu, Z. Li, and F. Tao, "Adaptive energy management strategy for hybrid batteries/supercapacitors electrical vehicle based on model prediction control," Asian J. Control, pp. 2463-2468, 2019, doi: 10.1002/asjc.2180.

28. O. Gomozov, J. P. F. Trovao, X. Kestelyn, and M. R. Dubois, “Adaptive Energy Management System Based on a Real-Time Model Predictive Control with Nonuniform Sampling Time for Multiple Energy Storage Electric Vehicle," IEEE Trans. Veh. Technol., vol. 66, no. 7, pp. 5520-5530, 2017, doi: 10.1109/TVT.2016.2638912.

29. P. Fortenbacher, J. L. Mathieu, and G. Andersson, “Modeling, identification, and optimal control of batteries for power system applications," Proc. - 2014 Power Syst. Comput. Conf. PSCC 2014, 2014, doi: 10.1109/PSCC.2014.7038360.

30. Z. Jiang and S. A. Raziei, "Hierarchical model predictive control for real-time energy-optimized operation of aerospace systems," AIAA Propuls. Energy Forum Expo. 2019, no. August, pp. 1-16, 2019, doi: 10.2514/6.2019-4487.

31. J. Seok, I. Kolmanovsky, and A. Girard, "Integrated / Coordinated Control of Aircraft Gas Turbine Engine and Electrical Power System : Towards Large Electrical Load Handling," IEEE 55th Conf. Decis. Control, no. Cdc, pp. 3183-3189, 2016.

32. J. P. S. Pinto Leite and M. Voskuijl, "Optimal energy management for hybrid-electric aircraft," Aircr. Eng. Aerosp. Technol., vol. 92, no. 6, pp. 851-861, 2020, doi: 10.1108/AEAT-03-2019-0046.

33. Y. Zhang et al., "An Energy Efficient Power Management Solution for a Fault-tolerant More Electric Engine/Aircraft," IEEE Trans. Ind. Electron., vol. PP, no. c, pp. 1-1, 2018, doi: 10.1109/TIE.2018.2877169.

34. M. Maasoumy, P. Nuzzo, F. Iandola, M. Kamgarpour, A. Sangiovanni-Vincentelli, and C. J. Tomlin, “Optimal load management system for Aircraft Electric Power distribution," IEEE Conf. Decis. Control, pp. 2939-2945, 2013, doi: 10.1109/CDC.2013.6760330.

35. X. Wang, J. Atkin, C. Hill, and S. Bozhko, "Power Allocation and Generator Sizing Optimisation of More-Electric Aircraft Onboard Electrical Power during Different Flight Stages," in AIAA 2019-4485 Session: Topic Area 3: System Optimization, pp. 1-10. doi: $10.2514 / 6.2019-4485$.

36. K. Ni et al., "Electrical and Electronic Technologies in More-Electric Aircraft: A Review,” IEEE Access, vol. 7, no. June, pp. 7614576166, 2019, doi: 10.1109/ACCESS.2019.2921622.

37. R. Seresinhe and C. Lawson, "Electrical load-sizing methodology to aid conceptual and preliminary design of large commercial aircraft," Proc. Inst. Mech. Eng. Part G J. Aerosp. Eng., vol. 229, no. 3, pp. 445-466, 2015, doi: 10.1177/0954410014534638.

38. A. Barzegar, R. Su, C. Wen, L. Rajabpour, Y. Zhang, and M. Y. Lee, "Intelligent Power Allocation and Load Management of More Electric Aircraft," Ieee Peds, no. June, pp. 533-538, 2015.

39. M. Elkazaz, M. Sumner, E. Naghiyev, S. Pholboon, R. Davies, and D. Thomas, "A hierarchical two-stage energy management for a home microgrid using model predictive and real-time controllers," Appl. Energy, vol. 269, no. April, p. 115118, 2020, doi: 10.1016/j.apenergy.2020.115118.

40. M. Madani, "OPL CPLEX tutorial : How to solve an optimization problem in IBM ILOG CPLEX Optimization studio".

41. G. Serale, M. Fiorentini, A. Capozzoli, D. Bernardini, and A. Bemporad, "Model Predictive Control (MPC) for enhancing building and HVAC system energy efficiency: Problem formulation, applications and opportunities," Energies, vol. 11, no. 3, 2018, doi: 10.3390/en11030631.

42. J. Garche and A. Jossen, “Battery management systems (BMS) for increasing battery life time," TELESCON 2000 - 3rd Int. Telecommun. Energy Spec. Conf. Proc., pp. 85-88, 2000, doi: 10.1109/TELESC.2000.918410.

43. X. Wang, Y. Gao, J. Atkin, and S. Bozhko, "Neural Network based Weighting Factor Selection of MPC for Optimal Battery and Load Management in MEA," 23rd Int. Conf. Electr. Mach. Syst. ICEMS 2020, pp. 1763-1768, 2020, doi: 10.23919/ICEMS50442.2020.9290968.

44. Y. Zhang, J. Chen, and Y. Yu, "Distributed power management with adaptive scheduling horizons for more electric aircraft," Int. J. Electr. Power Energy Syst., vol. 126, no. PA, p. 106581, 2021, doi: 10.1016/j.ijepes.2020.106581.

45. Australian Government Civil Aviation Safety Authority, "Aircraft Electrical Load Analysis and Power Source Capacity," 2017. 
46. M. Elkazaz, M. Sumner, and D. Thomas, "Real-time energy management for a small scale PV-battery microgrid: Modeling, design, and experimental verification," Energies, vol. 12, no. 14, 2019, doi: 10.3390/en12142713. 